\title{
Annual Performance of a Solar-Thermochemical Hydrogen Production Plant Based on $\mathrm{CeO}_{2}$ Redox Cycle
}

\author{
Alberto de la Calle $^{1} \quad$ Alicia Bayon $^{1}$ \\ ${ }^{1}$ CSIRO Energy, 10 Murray Dwyer Ct., Mayfield West, NSW 2304, Australia, \\ \{alberto.delacallealonso, alicia.bayonsandoval\}@csiro.au
}

\begin{abstract}
For the first time, a dynamic model of a 1-MW th thermochemical hydrogen production plant is developed and implemented for $\mathrm{CeO}_{2}$ redox cycle. The work explores how the variables of the process like the direct normal irradiation (DNI), temperature, pressure and degree of oxidation affect the annual production of hydrogen. The model reveals that the thermal inertia of $\mathrm{CeO}_{2}$ is significantly high to accomplish the oxidation without refrigerate the oxidizer. The operation is optimized to obtain the maximum amount of hydrogen in a year by only modifying the mass flow rates at the inlet of the reactors. The flexibility and adaptability of the model allows to test different system configurations and optimize the hydrogen production.
\end{abstract}

Keywords: Solar fuels, Central receiver, High temperature, Dynamic modelling

\section{Introduction}

Solar energy is, by far, able to be massively exploited for delivering all of the world energy needs utilizing only a few percent of the deserted areas (IRENA and IEA-ETSAP, 2013; Lewis and Nocera, 2007). Nevertheless, the storage of the thermal energy for its use during the non-solar periods is required to couple production/demand rate in the energy market. In this context, the conversion of the solar concentrated source into storable and transportable fuels is a remarkable alternative to extent the commercialization of solar power technologies.

One attractive pathway is the solar thermal production of hydrogen. Within all possible solar driven routes, solar thermochemical $\mathrm{H}_{2} \mathrm{O}$ splitting offers a path to produce carbon-free hydrogen. Hydrogen is an energy carrier in addition to a commodity used for the several industrial processes (Ramachandran and Menon, 1998). Nevertheless, direct thermolysis of $\mathrm{H}_{2} \mathrm{O}$ requires temperatures well above $2000 \mathrm{~K}$ to obtain significant $\mathrm{H}_{2}$ concentrations (Fletcher, 2001). In addition, to avoid the recombination of the product gas $\mathrm{H}_{2}$ and $\mathrm{O}_{2}$ upon cooling, they need to be separated at the dissociation temperature, which is technically challenging (Fletcher, 2001). In this respect, $\mathrm{H}_{2} \mathrm{O}$-splitting thermochemical cycles have been investigated to reduce the process operating temperature compared to direct thermolysis. In addition, the need for high-temperature product gas separation is eliminated, because $\mathrm{H}_{2}$ and $\mathrm{O}_{2}$ are produced in separate process steps. Compared to multi-step cycles, two-step cycles promise to reach higher process efficiencies due to higher operating temperatures and less irreversibilities (Abanades et al., 2006).

Besides the environmental benefits of the thermochemical cycles, several impediments must be confronted to the economic realization which concerns the design of reactor to reduce the radiation and conduction losses and materials development revealing satisfactory durability, reactivity and efficiencies (D'Souza, 2013; Roeb et al., 2012). Likewise, heat and mass transfer play a crucial role in the building components and for the technological implementation of thermochemical reactors.

Up to date, 300 redox systems have been proposed although only few tens of them have been performed experimentally mainly due to temperature and thermodynamic limitations (Muhich et al., 2015). In terms of economic assessment, a recent report has indicated that solar fuels produced with $20 \%$ efficiency are likely to be cost competitive (Kim et al., 2012). Upon all the possible metal oxide candidates, Ceria $\left(\mathrm{CeO}_{2}\right)$ is the most promising material so far studied during the last 50 years because it demonstrates faster hydrogen production kinetics and high selectivity (Ackermann and Steinfeld, 2014; Chueh et al., 2010; Furler et al., 2012; Gao et al., 2016; Scheffe and Steinfeld, 2012). In this cycle, the nonstoichiometric ceria, with fluorite-type structure, retain the oxygen vacancies maintaining its cyclability. The reactions involved in this process are:

$$
\begin{gathered}
\mathrm{CeO}_{2} \rightarrow \mathrm{CeO}_{2-\delta}+\frac{\delta}{2} \mathrm{O}_{2}(\mathrm{~g}) \\
\mathrm{CeO}_{2-\delta}+\delta \mathrm{H}_{2} \mathrm{O}(\mathrm{g}) \rightarrow \mathrm{CeO}_{2}+\delta \mathrm{H}_{2}(\mathrm{~g})
\end{gathered}
$$

The thermal reduction (Equation 1) occurs at temperatures not lower than $1500{ }^{\circ} \mathrm{C}$ accompanied by a low $\mathrm{O}_{2}$ partial pressure about $1 \mathrm{~Pa}$ (Chueh et al., 2010). This condition requires a large amount of inert gas flowing into the reaction media and, consequently, an enormous economic penalty influenced by three factors: cost of inert gas, separation of $\mathrm{O}_{2}$ produced downstream and energy losses transferred to the gas (Furler et al., 2012). In the low temperature step, the exothermic water splitting (Equation 2) 
takes place at lower temperatures commonly between 600 and $1000{ }^{\circ} \mathrm{C}$.

The thermochemical efficiency was largely explored in previous works (Bader et al., 2013; Ermanoski, 2015; Ermanoski et al., 2013; Bulfin et al., 2015). In this context, a maximum of $68 \%$ could be obtained if all the $\mathrm{CeO}_{2}$ is reduced to $\mathrm{Ce}_{2} \mathrm{O}_{3}$ at $2200{ }^{\circ} \mathrm{C}$ with the sun as only heat source. However, at $1500{ }^{\circ} \mathrm{C}$ only $2 \%$ conversion is obtained in real conditions, lowering the efficiency up to $1.72 \%$ from solar to fuel without heat recovery (Furler et al., 2012). This value could be enhanced to $20 \%$ if ideal heat recovery is applied (Ermanoski et al., 2013). However, all the previous thermodynamic analysis are based on steady-state simulations with the aim of maximizing the reactor efficiency of the process giving an single value of DNI without considering the variability of the solar resource, heliostat field design and receiver performance. The goal of this work is to provide insights on the effect of the variability of the solar resource over the annual performance emulating a solar production plant based on a Ceria thermochemical water splitting cycle.

In the present paper, a new dynamic model of a solar hydrogen production plant is developed for annual simulations. The model is based on an object-oriented modelling methodology following a modular and hierarchical structure. The final model has been graphically implemented by connecting different components which encapsulate the main thermodynamic processes that take place in the plant. Modelica and Dymola 2017 were the language and the simulation environment used in this work.

\section{System description}

A 1-MW $\mathrm{M}_{\text {th }}$ solar hydrogen plant is designed to be placed in Geraldton (WA), Australia. Table 1 shows the system design specifications. It consists of two rotatory reactors (for reduction and oxidation), where a flow of particles of $\mathrm{CeO}_{2}$ is recirculated in order to efficiently use the thermal inertia of the reactors.

The thermal reduction (Equation 1) is endothermic and takes place in a windowed reactor-receiver where the concentrated solar radiation directly heats the moving bed of particles. Bader et al. (2013) suggests a concentration ratio of 3000 to get a high efficiency ratio according the following equation:

$$
\dot{Q}_{s o l, 0}=A_{\text {rea }} C_{0} G_{0},
$$

The design parameters are defined as follows: $\dot{Q}_{s o l, 0}=10^{6} \mathrm{~W}$ which is the design power at the receiver, $G_{0}=950 \mathrm{~W} / \mathrm{m}^{2}$ that is the DNI and $C_{0}=3000$ which is the concentration ratio. This expression allows to obtain the diameter of the aperture (considered circular) of the receiver at the design point $(0.67 \mathrm{~m})$.

In order to get a suitable concentration ratio at the receiver aperture, a secondary concentrator is placed to increase the flux density of the radiation. A compound parabolic concentrator (CPC) has demonstrated high performance in this kind of processes (Pitz-Paal et al., 2011).
Table 1. System design specification.

\begin{tabular}{ll}
\hline \multicolumn{2}{c}{ Solar resource } \\
Location: & Geraldton (WA) \\
Longitude: & $114.7^{\circ}$ \\
Latitude: & $-28.8^{\circ}$ \\
Local time zone: & $\mathrm{UCT}+8$ \\
\multicolumn{2}{c}{ Heliostat field } \\
Heliostat size: & $2.44 \mathrm{x} 1.84 \mathrm{~m}$ \\
Number of heliostats: & 604 \\
Mirror reflectivity: & 0.95 \\
Soiling factor: & 0.95 \\
Heliostat availability: & 0.99 \\
\multicolumn{2}{c}{ Solar tower } \\
Design thermal power: & $1 \mathrm{MW}$ \\
DNI design value: & $950 \mathrm{~W}$ \\
Tower height: & $19.45 \mathrm{~m}$ \\
Receiver elevation: & $-10^{\circ}$ \\
Receiver acceptance angle: & $70^{\circ}$ \\
CPC aperture diameter: & $1.16 \mathrm{~m}$ \\
Reactor aperture diameter: & $0.67 \mathrm{~m}$ \\
Flux shape factor: & 0.87 \\
Solar concentration ratio: & 3000 \\
\hline
\end{tabular}

These devices, based on non-imaging optics, collect radiation entering the entrance aperture diameter $\left(D_{C P C}\right)$ within angle of $\theta_{C P C}$ and direct it to the reactor aperture diameter $\left(D_{\text {rea }}\right)$ with negigible losses (O'Gallagher and Winston, 1983). The relationship between the aperture angle and the concentration ratio is:

$$
C_{C P C}=\frac{1}{\sin ^{2}\left(\theta_{C P C}\right)},
$$

and the relationship between both (CPC and reactor) aperture diameters is:

$$
D_{C P C}=\frac{D_{r e a}}{\sin \left(\theta_{C P C}\right)} .
$$

The typical values of the heliostat field concentrating ratio rounds 1000 . In this respect, a value of 3 for the CPC concentrating ratio is required to provide the required design parameters. According this value, the acceptance angle (i.e. $2 \theta_{C P C}$ ) is $70^{\circ}$ and the CPC diameter $1.16 \mathrm{~m}$.

SolarPILOT $^{T M}$ (NREL, 2016) was used to design and optimize the heliostat field. It allows fast generation and optimization of solar fields according a series of design parameters. Figure 1 shows the optimized solar field layout for this study. In addition, SolarPILOT ${ }^{T M}$ provides the total optical efficiency of the solar field which includes cosine error, reflectivity and soiling, blocking and shading, atmospheric attenuation and scattering and spillage of a whole year as function of the zenith and azimuth solar angles (Table 2). The efficiency factor is calculated for a specific receiver, in this case, a $1.16 \mathrm{~m}$ side-squared receiver. In order to compensate the difference between 
Table 2. Reference solar field optical efficency as a function of Zenith and Azimuth angles.

\begin{tabular}{ccccccccccccccc}
\hline$\theta_{z e n} \backslash \theta_{\text {azi }}$ & $-150^{\circ}$ & $-120^{\circ}$ & $-90^{\circ}$ & $-60^{\circ}$ & $-30^{\circ}$ & $0^{\circ}$ & $30^{\circ}$ & $60^{\circ}$ & $90^{\circ}$ & $120^{\circ}$ & $150^{\circ}$ & $180^{\circ}$ \\
$0.5^{\circ}$ & 0.42947 & 0.42832 & 0.41992 & 0.40721 & 0.39708 & 0.39435 & 0.39443 & 0.39397 & 0.39521 & 0.40576 & 0.41858 & 0.42729 \\
$7^{\circ}$ & 0.37594 & 0.38236 & 0.39179 & 0.40135 & 0.40839 & 0.41095 & 0.40835 & 0.40135 & 0.39176 & 0.38262 & 0.37587 & 0.37355 \\
$15^{\circ}$ & 0.35315 & 0.36675 & 0.38640 & 0.40662 & 0.42085 & 0.42596 & 0.42087 & 0.40617 & 0.38656 & 0.36641 & 0.35311 & 0.34833 \\
$30^{\circ}$ & 0.30117 & 0.33196 & 0.37561 & 0.41417 & 0.43945 & 0.44756 & 0.43918 & 0.41437 & 0.37532 & 0.33190 & 0.30110 & 0.29084 \\
$45^{\circ}$ & 0.25081 & 0.30291 & 0.36867 & 0.41824 & 0.44617 & 0.45552 & 0.44604 & 0.41737 & 0.36826 & 0.30301 & 0.25063 & 0.23260 \\
$60^{\circ}$ & 0.20337 & 0.26952 & 0.34444 & 0.39892 & 0.43285 & 0.44481 & 0.43247 & 0.39877 & 0.34452 & 0.26885 & 0.20281 & 0.16978 \\
$75^{\circ}$ & 0.15311 & 0.20778 & 0.27510 & 0.33189 & 0.37052 & 0.37133 & 0.37056 & 0.33101 & 0.27444 & 0.20749 & 0.15253 & 0.11308 \\
$85^{\circ}$ & 0.09236 & 0.11869 & 0.14569 & 0.14285 & 0.15079 & 0.14289 & 0.15139 & 0.14214 & 0.14519 & 0.11818 & 0.09174 & 0.06352 \\
\hline
\end{tabular}

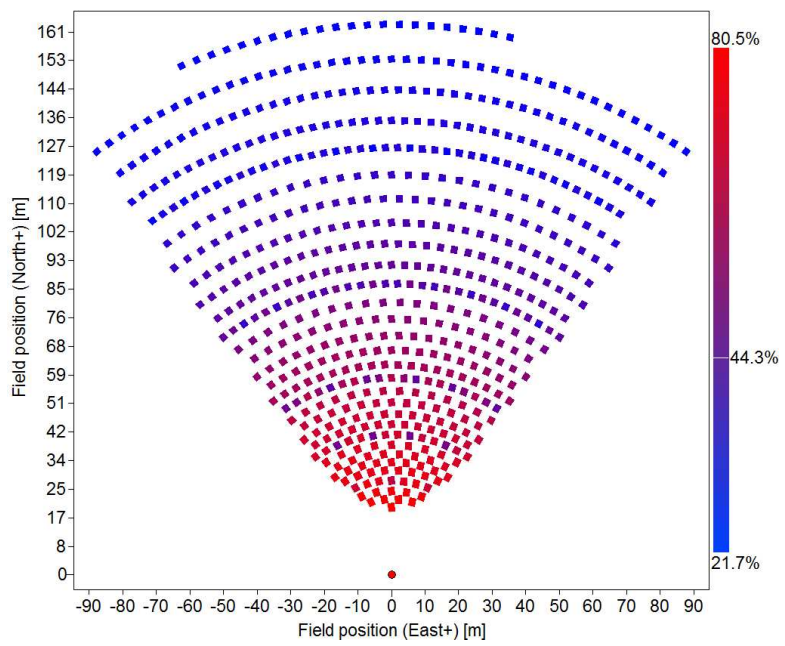

Figure 1. Heliostat field layout.

both shapes, square and circle, a correction factor is applied. This shape factor, that is the fraction of the total concentrated power in both shapes, has a value of 0.87 .

The oxygen generated during the reduction of $\mathrm{CeO}_{2}$ should be removed in order to get an optimum reduction performance. $\mathrm{O}_{2}$ is pushed out by a purge flow of high purity $\mathrm{N}_{2}$ allowing reach a very low oxygen partial pressure inside the reactor.

The hydrogen production is accomplished at the oxidizer and depends on the temperature, the reduction degree of the moving particles of $\mathrm{CeO}_{2}$ and the amount of water entering the reactor. In order to obtain a high production of hydrogen, this plant considers $\mathrm{CeO}_{2-\delta}$ as the limiting reagent (Equation 2). It is expected that the residence time of the $\mathrm{CeO}_{2-\delta}$ inside the oxidizer is sufficient to achieve the complete oxidation. A small tank of $\mathrm{CeO}_{2}$ after the oxidizer allows a better management of the $\mathrm{CeO}_{2}$ particles in the cycle.

In order to achieve a higher system efficiency, several heat recovery strategies were implemented. Two shelland-tubes heat exchangers placed at the input of both reactors to recover the sensible heat of the gases. Furthermore, it is assumed that steam lines are pre-heated up to 200 ${ }^{\circ} \mathrm{C}$ in order to prevent condensation. Finally, a solid-solid heat exchanger is placed between both reactors (receiver and oxidiser) to recover the sensible heat of $\mathrm{CeO}_{2}$ particles exiting the receiver as proposed in previous works (Bader et al., 2013; Ermanoski, 2015; Ermanoski et al.,
2013; Bulfin et al., 2015).

\section{Object-oriented modelling}

The model described in this section follows an objectoriented methodology based on equations. The main physical and chemical phenomena were identified and encapsulated into independent and reusable modules. These modules are connected creating hierarchical structures. This approach allows to study different plant configuration to improve the annual performance.

The model was implemented in Modelica language (Modelica Association, 2016) and is fully compatible with Modelica Standard Library (MSL). Modelica Fluid and Modelica Thermal connectors were used to define relationships between components. The thermodynamic properties of fluids are obtained from medium models that extend from Modelica Media Interface (MMI). All the submodels are locally balanced ensuring robust modelling and debugging (Olsson et al., 2008).

\subsection{Subsystem modelling}

The system model that reproduces the plant described in $\S 2$, is presented in Figure 2. It consist on the following sub-models: data source, sun, heliostat field, receiver, oxidizer, tank, heat exchangers, pumps and valve. This model also includes: fluid source, fluid boundary, thermal source, real expression and medium sub-models.

General assumptions are summarized as follows:

- $\mathrm{CeO}_{2}$ particle properties are assumed to be quasifluid.

- One-dimensional consideration within the direction of heat and mass flows.

- Heat conduction and radiation are negligible in fluid components. Axial heat flow is also negligible in both fluids.

- Lumped thermodynamic properties are assumed in fluid components.

- Chemical reactions only take place in receiver and oxidizer.

Receiver and oxidizer sub-models are fully described with complete set of equations in this work. The remaining models were obtained and adapted from exist- 


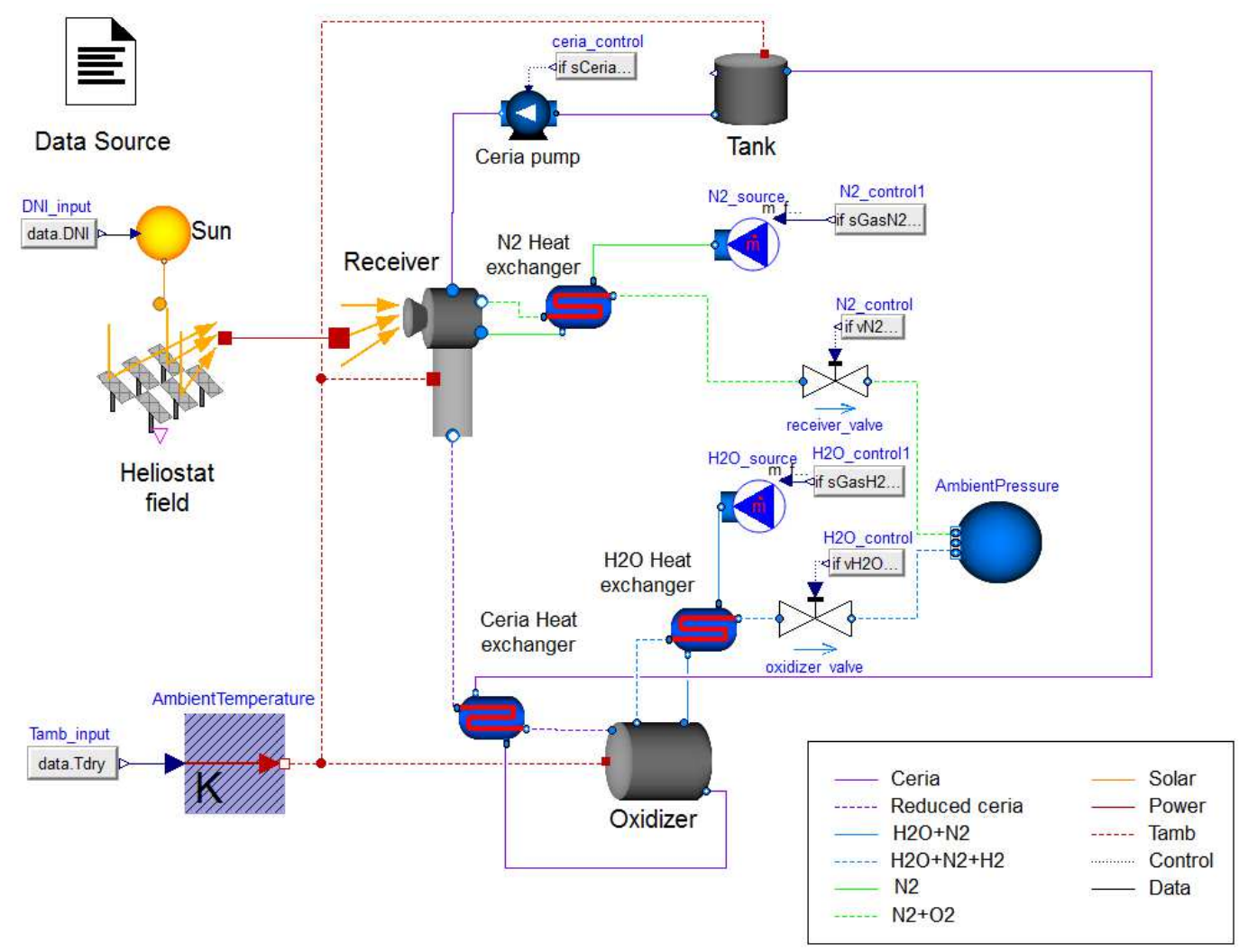

Figure 2. Modelica component diagram of the system model.

ing libraries. In fact, this demonstrates the high reusability, extensibility and customizability of the modelling methodology used. The models of data source, sun, heliostat field and tank are been re-used from the opensource SolarTherm library (de la Calle et al., 2016a) with some adaptations and extensions. This library is available at https://github.com/solartherm/solartherm and consists on concentrating solar thermal (CST) components that are used to perform the annual simulations and the economic assessments of solar thermal plants. The models of heat exchangers and pumps are utilized and adapted from previous works of de la Calle et al. (2016b). The models of fluid source, fluid boundary, linear pressure drop valve, thermal source and real expression are included on the MSL and medium models extends from MMI.

A brief description of each one of the sub-models is provided below:

\subsubsection{Medium models}

Two medium models were implemented to describe gas mixture and Ceria properties. The gas medium is used in both reactors and extends from the Modelica Media IdealGases.Common.MixtureGasNasa. This medium is composed of water, oxygen, hydrogen and nitrogen at its gaseous state and assumes ideal gas properties provided by McBride et al. (2002).

The ceria medium model includes a function of the degree of reduction $(\delta)$ explicit in temperature and oxygen partial pressure (Ermanoski et al., 2013). In addition, the medium includes a function for knowing the minimum required amount of water to achieve the oxidation based on the water equilibrium and heat of reaction explicit in $\delta$ (Bulfin et al., 2015).

\subsubsection{Data source}

This model encapsulates the extraction of weather data. It uses a MSL's CombitimeTable with spline interpolation such that derivatives are continuous. The raw file is a typical meteorological year data set in the TMY3-file format (Wilcox and Marion, 2008). In order to be readable, the file is modified being compatible with Modelica specifications (Modelica Association, 2016).

\subsubsection{Sun}

This model provides the sun position relative to the plant location and the DNI in every time step. Users can choose between different correlations such as Duffie and Beckman (2013) or Blanco-Muriel et al. (2001) for calculating the declination and solar hour angles. The time variable matches with the local time where $0 \mathrm{~s}$ is 00:00 of 1st of January in this time zone. The DNI is provided by a RealInput connector.

\subsubsection{Heliostat field}

This model calculates the total concentrated solar power of the heliostat field $\left(\dot{Q}_{\text {sol }}\right)$ as:

$$
\dot{Q}_{s o l}=N_{h e l} A_{h e l} \eta_{a v} \eta_{o p} G
$$


where the number of heliostats $\left(N_{\text {hel }}\right)$, the heliostat area $\left(A_{\text {hel }}\right)$ and the heliostat availability $\left(\eta_{a v}\right)$ are design parameters (Table 1). Solar angles and DNI $(G)$ are provided by the SolarPort connector and the total optical efficiency $\left(\eta_{o p}\right)$ is calculated using the MSL's Combitable2D with spline interpolation and the Table 2 as input. The start-up and the shutdown of the plant is automatically controlled according the minimum starting power, minimum operating power and the deploy angle, which are model parameters (Appendix I).

\subsubsection{Receiver}

This model provides the dynamic amount of $\mathrm{CeO}_{2}$ reduced at the reactor. It is designed to perform annual simulations, therefore it is able to deal with zero-mass flow rates and zero mass. It is a lumped parameter model which assumes a single control volume (CV). The main particular assumptions are the following:

- Infinite thermal conductivity inside the reactor: same temperature at shell, $\mathrm{CeO}_{2}$ and gas.

- Black body receiver approach: while radiative thermal losses are considered only at the reactor aperture, convective thermal losses are considered at all the external reactor surface.

- Perfect mixer approach: both inner $\mathrm{CeO}_{2}$ particles and gas are perfectly mixed with their respectively accumulated masses.

- Pressure drop is neglected inside the reactor. The same pressure is assumed in all the $\mathrm{CV}$.

- Constant inner molar flow rate of $\mathrm{CeO}_{2}$ is assumed. The residence time of $\mathrm{CeO}_{2}$ inside the reactor is constant.

The gas mass inside of the reactor $\left(m_{g}\right)$ is determined by the inner gas $\left(\dot{m}_{g, \text { in }}\right)$, the outer gas $\left(\dot{m}_{g, \text { out }}\right)$ and the gas produced during the reduction $\left(\dot{m}_{g e n, O_{2}}\right)$ :

$$
\dot{\boldsymbol{m}}_{g}=\dot{m}_{g, \text { in }}-\dot{m}_{g, \text { out }}+\dot{m}_{g e n, O_{2}} .
$$

The gas pressure $(p)$ is determined by means of the ideal gas law:

$$
p V_{g}=m_{g} k_{g} T,
$$

where the specific gas constant $\left(k_{g}\right)$ depends on the mass fraction of gases. The volume of the reactor $(V)$ is constant and filled with $\mathrm{CeO}_{2}\left(V_{c e}\right)$ and gas $\left(V_{g}\right)$ :

$$
V=V_{g}+V_{c e} .
$$

The oxygen mass balance is calculated according the mass fractions:

$$
\dot{\boldsymbol{m}}_{g, O_{2}}=\dot{m}_{g, i n} X_{O_{2}, \text { in }}-\dot{m}_{g, \text { out }} X_{O_{2}}+\dot{m}_{g e n, O_{2}} .
$$

The outer mass fraction is the same as the CV mass fraction:

$$
\begin{aligned}
X_{O_{2}} & =\max \left(0, \frac{m_{g, O_{2}}}{m_{g}}\right), \\
X_{H_{2} O} & =1-X_{O_{2}},
\end{aligned}
$$

where the maximum function is used to avoid numerical problems. The oxygen generated during the reduction is calculated as function of the oxygen molecular mass $\left(\mathrm{M}_{\mathrm{O}_{2}}\right)$ and the generated molar flow $\left(\dot{n}_{g e n, \mathrm{O}_{2}}\right)$ :

$$
\dot{m}_{g e n, O_{2}}=M_{O_{2}} \dot{n}_{g e n, O_{2}} .
$$

The generated molar flow depends on the degree of reduction $(\delta)$ and the inner $\mathrm{CeO}_{2}$ molar flow $\left(\dot{n}_{c e, i n}\right)$ :

$$
\dot{n}_{g e n, O_{2}}=\frac{\dot{n}_{c e, i n} \delta}{2} .
$$

The amount of $\mathrm{CeO}_{2}\left(n_{c e}\right)$ inside the reactor is calculated by the molar balance:

$$
\dot{\boldsymbol{n}}_{c e}=\dot{n}_{c e, \text { in }}-\dot{n}_{c e, \text { out }},
$$

where the molar flow at outlet $\left(\dot{n}_{c e, \text { out }}\right)$ is calculated according the following when-clause:

$$
\dot{n}_{c e, \text { out }}= \begin{cases}\dot{n}_{c e, \text { in }} & \text { when } n_{c e} \geq n_{c e, \max }, \\ 0 & \text { elsewhen } n_{c e} \leq 0\end{cases}
$$

The maximum number of moles $\left(n_{c e, \max }\right)$ is calculated according the maximum volume of $\mathrm{CeO}_{2}$ which is a model parameter (Appendix I).

The mass of $\mathrm{CeO}_{2}\left(m_{c e}\right)$ is determined by its molar mass $\left(M_{c e}\right)$ which depends on delta:

$$
m_{c e}=M_{c e} n_{c e},
$$

and the volume by:

$$
V_{c e}=\frac{m_{c e}}{\rho_{c e}} .
$$

$\delta$ is calculated as function of the temperature and the partial pressure of oxygen $\left(p_{\mathrm{O}_{2}}\right)$. The maximum value of $\delta$ is limited to 0.25 which is the maximum value possible to maintain the fluorite structure of $\mathrm{CeO}_{2}$. The partial pressure is calculated as:

$$
p_{O_{2}}=\frac{m_{O_{2}}}{M_{O_{2}}} p .
$$

The temperature of the reactor is calculated according to the global energy balance:

$$
\eta_{s h} \dot{Q}_{s o l}-\dot{Q}_{l o s s}=\Delta \dot{Q}_{r e}+\Delta \dot{Q}_{g}+\Delta \dot{Q}_{c e},
$$

where the concentrated solar power $\left(\dot{Q}_{s o l}\right)$ coming from the heliostat field is attenuated by the shape factor $\left(\eta_{s h}\right)$. 
The heat loss $\left(\dot{Q}_{\text {loss }}\right)$ is the sum of the radiative $\left(\dot{Q}_{\text {loss }, r d}\right)$ and convective losses $\left(\dot{Q}_{l o s s, r d}\right)$ :

$$
\begin{aligned}
& \dot{Q}_{\text {loss }, r d}=A_{a p} \sigma \xi\left(T^{4}-T_{a m b}^{4}\right), \\
& \dot{Q}_{l o s s, c v}=A_{r e} \alpha\left(T-T_{a m b}\right) .
\end{aligned}
$$

The radiative losses are only applicable to the aperture area of the reactor due to the lack of thermal insulation, and the convective losses to the whole reactor. Emissivity $(\xi)$ and heat transfer coefficient $(\alpha)$ are model parameters and the ambient temperature $\left(T_{a m b}\right)$ is an input of the model.

The receiver mass contribution into the energy balance $\left(\Delta \dot{Q}_{r e}\right)$ is mostly due to the thermal inertia of the metal cover:

$$
\Delta \dot{Q}_{r e}=m_{r e} C_{p, r e} \dot{\boldsymbol{T}},
$$

where the mass $\left(m_{r e}\right)$ and the specific heat capacity $\left(C_{p, r e}\right)$ are model parameters. The gas contribution into the energy balance is determined by:

$$
\Delta \dot{Q}_{g}=m_{g} C_{p, g} \dot{\boldsymbol{T}}+\dot{m}_{g, i n}\left(h_{g}-h_{g, i n}\right) .
$$

where it is assumed that the outlet temperature is the same as the temperature inside the reactor. The following equation calculates the $\mathrm{CeO}_{2}$ contribution into the energy balance:

$$
\Delta \dot{Q}_{c e}=m_{c e} C_{p, c e} \dot{\boldsymbol{T}}+\dot{m}_{c e, i n}\left(h_{c e}-h_{c e, i n}\right)-n_{c e, i n} \delta Q_{r e d} .
$$

where it is also assumed that the outlet temperature is the same as the temperature inside the reactor and heat of reduction $\left(Q_{\text {red }}\right)$ depends on $\delta$.

\subsubsection{Oxidizer}

This model dynamically provides the amount of hydrogen produced. It is a lumped parameter model $(1 \mathrm{CV})$ similar to the receiver. The particular assumptions are the same as for the receiver but in this case, due to the lack of an aperture, only convection losses are been considered. The main assumption is the complete oxidation of the $\mathrm{CeO}_{2}$.

The gas mass inside of the reactor is determined by the inflowing gas, the outflowing gas, the gas produced $\left(\dot{m}_{g e n, \mathrm{H}_{2}}\right)$ and the consumed at the oxidation $\left(\dot{m}_{\text {con }, \mathrm{H}_{2} \mathrm{O}}\right)$ :

$$
\dot{\boldsymbol{m}}_{g}=\dot{m}_{g, \text { in }}-\dot{m}_{g, \text { out }}+\dot{m}_{g e n, \mathrm{H}_{2}}-\dot{m}_{c o n, \mathrm{H}_{2} \mathrm{O}} .
$$

The oxidizer pressure and the volume calculation are determined by Equations 8 and 9 .

The mass balances of nitrogen, hydrogen and water are calculated according the mass fractions:

$$
\begin{aligned}
\dot{\boldsymbol{m}}_{g, \mathrm{~N}_{2}} & =\dot{m}_{g, \text { in }} X_{N_{2}, \text { in }}-\dot{m}_{g, \text { out }} X_{N_{2}}, \\
\dot{\boldsymbol{m}}_{g, H_{2}} & =\dot{m}_{g, \text { in }} X_{\mathrm{H}_{2}, \text { in }}-\dot{m}_{g, \text { out }} X_{\mathrm{H}_{2}}+\dot{m}_{g e n, H_{2}}, \\
\dot{\boldsymbol{m}}_{g, H_{2} \mathrm{O}} & =\dot{m}_{g, \text { in }} X_{\mathrm{H}_{2} \mathrm{O}, \text { in }}-\dot{m}_{g, \text { out }} X_{\mathrm{H}_{2} \mathrm{O}}-\dot{m}_{\text {con }, H_{2} O} \mathrm{O}
\end{aligned}
$$

The outflowing mass fractions are determined by:

$$
\begin{aligned}
X_{N_{2}} & =\min \left(1, \frac{m_{g, N_{2}}}{m_{g}}\right), \\
X_{H_{2}} & =\max \left(0, \frac{m_{g, H_{2}}}{m_{g}}\right), \\
X_{H_{2} O} & =\min \left(1, \frac{m_{g, H_{2} O} O}{m_{g}}\right),
\end{aligned}
$$

where maximum and minimum functions are used for preventing numerical problems.

The mass flows due to the oxidation are:

$$
\dot{m}_{g e n, \mathrm{H}_{2}}=M_{\mathrm{H}_{2}} \dot{n}_{g e n, \mathrm{H}_{2}}, \dot{m}_{\mathrm{con}, \mathrm{H}_{2} \mathrm{O}}=\mathrm{M}_{\mathrm{H}_{2} \mathrm{O}} \dot{n}_{\text {con } \mathrm{H}_{2} \mathrm{O}},
$$

where the molar flows depends on the degree of reduction of the inflowing $\mathrm{CeO}_{2}$ :

$$
\begin{aligned}
\dot{n}_{c o n, \mathrm{H}_{2} \mathrm{O}} & =\frac{\dot{n}_{c e, i n} \delta}{2}, \\
\dot{n}_{g e n, \mathrm{H}_{2}} & =\dot{n}_{c o n, \mathrm{H}_{2} \mathrm{O}} .
\end{aligned}
$$

Equations 15-18 are used in this model to calculate the $\mathrm{CeO}_{2}$ mass and volume dynamics. The temperature is calculated according to the global energy balance:

$$
-\dot{Q}_{\text {loss }, c v}=\Delta \dot{Q}_{r e}+\Delta \dot{Q}_{g}+\Delta \dot{Q}_{c e},
$$

where Equations $22-24$ provide the heat losses, the receiver mass contribution and the gas contribution to the energy balance. The $\mathrm{CeO}_{2}$ contribution is determined by:

$$
\Delta \dot{Q}_{c e}=m_{c e} C_{p, c e} \dot{\boldsymbol{T}}+\dot{m}_{c e, i n}\left(h_{c e}-h_{c e, i n}\right)-n_{c e, i n} \delta Q_{o x, c e} .
$$

where heat of oxidation is assumed as $Q_{o x}=-Q_{\text {red }}$.

\subsubsection{Tank}

This model introduces the dynamics of a small storage element which pressure is fixed parametrically. It is a lumped parameter model which assumes a cylinder volume and an ideally mixed fluid. The mass balance is:

$$
\dot{\boldsymbol{m}}=\dot{m}_{\text {in }}-\dot{m}_{\text {out }},
$$

and the energy balance is:

$$
m \dot{\boldsymbol{h}}=\dot{m}_{\text {in }}\left(h_{\text {in }}-h\right)-\dot{Q}_{\text {loss }},
$$

where shell capacitance is neglected. The convective heat losses to the environment are only applied to the metal surface that is in contact with the fluid.

\subsubsection{Heat exchanger}

This quasi-steady-state heat exchanger model allows to calculate sensible heat transfer between two fluids based on the mathematical development of Spakovszky (2008) and whose implementation was performed by de la Calle et al. (2016b). It is a lumped parameter model which assumes that every state at the heat exchanger is locally steady. The model is able to manage zero-mass flow rates. 


\subsubsection{Pump}

This ideal pump model provides a controlled mass flow rate between two points in the same streamline.

\subsubsection{Linear valve}

This model calculates the mass flow rate that crosses through the valve opening with a linear approximation of the pressure drop.

\subsubsection{Fluid source}

This model provides an input boundary condition where the mass flow rate, the specific enthalpy and mass fraction are defined. It is used to simulate the inflowing gas at the reactors.

\subsubsection{Fluid boundary}

This model provides an output boundary condition where the pressure, the specific enthalpy and mass fraction are defined. It is used to simulate the environment (fluid models).

\subsubsection{Thermal source}

This model provides a thermal boundary condition where the temperature is defined. It is used to simulate the environment (thermal models).

\subsubsection{Real expression}

It is a model used to connect experimental data as inputs of the models in a graphical way.

\subsection{Automatic control system}

The automatic control system (ACS) is designed to guarantee the stability of the plant in annual simulations. This system is made up by a series of on-off controllers which control the circulation of the fluids inside the plant.

The ACS must prevent the reverse flow of gases at valves. For this reason, the valves are only opened when the pressure drop is higher than half of the nominal pressure drop.

The gas source is opened since the heliostat field reaches the start-up power and it is closed when the heliostat field is shut down and the reactor temperatures are below a certain shutdown temperature.

The ceria pump starts when the heliostat field is started, both gas valves are open and the receiver temperature is higher than a minimum operating temperature. In order to take advantage of the thermal inertia of the reactors, the pump shut down when the heliostat field is shut down and the receiver temperature is below the minimum operating temperature.

\section{Simulation}

Dymola 2017 (Dassault Systemes, 2016) was the tool used for the Modelica implementations and simulations. The numerical solver used for the dynamic simulations has been DASSL (Petzold, 1983) whose absolute and relative tolerances were set to $10^{-4}$.
The model is a set of high-index differential and algebraic equations (DAEs) of 745 scalar variables. After the translation, the model has 255 time-varying variables and 14 continuous-time states.

The numerical value of model parameters can be reviewed in Appendix I. The annual performance of the plant is very sensible to the operating parameters. Few operating parameters have been optimised: $\mathrm{CeO}_{2}$ mass flow rate, inlet receiver gas flow rate and inlet oxidizer mass flow rate and its composition. The optimization method was the Simplex algorithm and the objective function was the final hydrogen production.

The annual simulation was performed using weather data for the Geraldton location provided by AUSTELA (2016). The CPU-time for integration was $161 \mathrm{~s}$ for the whole year simulated with 9997 state events mostly related with the ACS.

The amount of hydrogen produced during the simulated year is $46.57 \mathrm{t}$. The variation in time of this production is depicted in Figure 3, where the different seasonal rate (winter-summer) can be observed. The solar to hydrogen efficiency of the plant, defined as:

$$
\eta=\frac{m_{H_{2}} H H V}{Q_{s o l}}
$$

where $m_{\mathrm{H}_{2}}$ is the annual amount of hydrogen, $H H V$ is the hydrogen heating value and $Q_{s o l}$ is the annual amount of energy that reach the heliostat field, has a value of $25.27 \%$. This result is in line with previous works (Bader et al., 2013; Ermanoski, 2015; Ermanoski et al., 2013; Bulfin et al., 2015).

Figure 4 shows the simulation details of 5 days (from 28 August to 1 September). This week has one sunny day (240), three partially cloudy days (241, 243 and 244) and one completely cloudy day (242) (Figure 4(a)). The concentrated solar power by the heliostat field is shown in Figure 4(b). The plant does not use all the available energy $\left(\dot{Q}_{\text {raw }}\right)$ because a minimum start-up power and minimum operating power are applied. Although 1.2 MW of peak power is reached 2 days, only few hour per day the power is higher than $1 \mathrm{MW}$. At the day 242, the system did not achieve the start-up power in the whole day and at

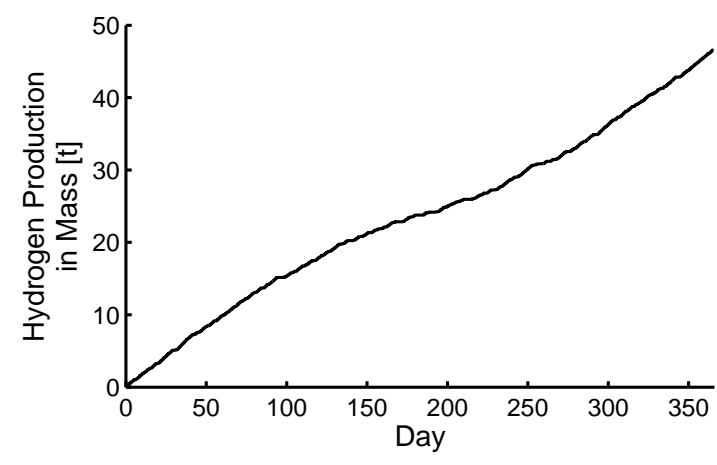

Figure 3. Annual hydrogen production. 


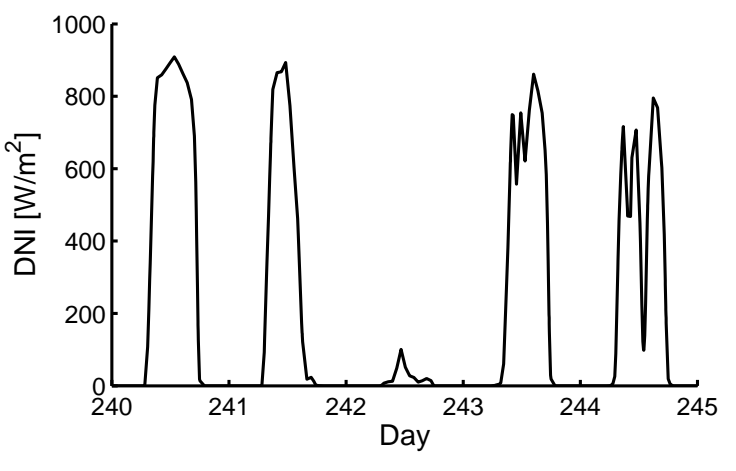

(a) Direct normal radiation.

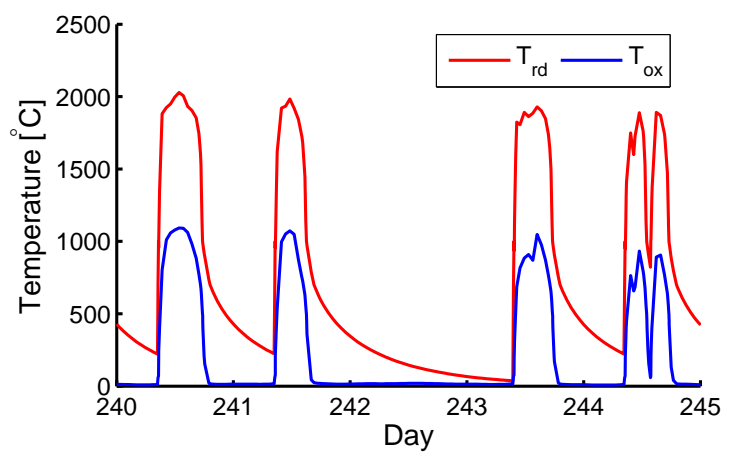

(c) Receiver and oxidizer temperatures.

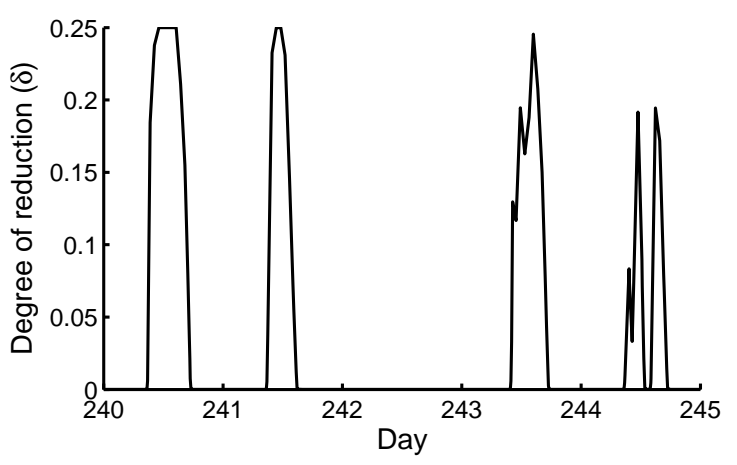

(e) Degree of reduction.

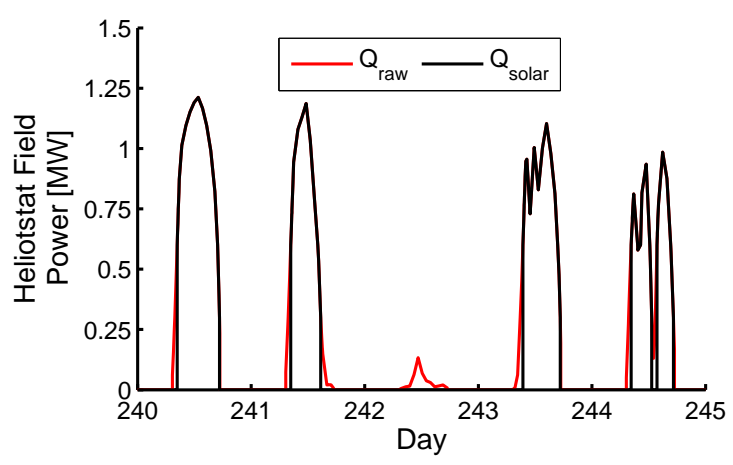

(b) Heliostat field power.

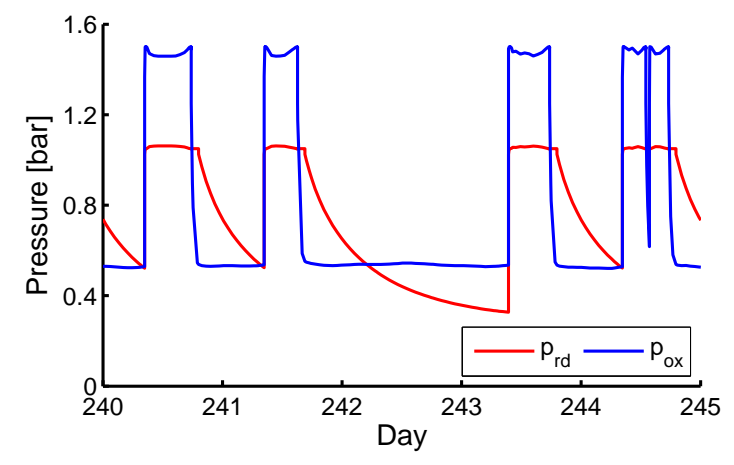

(d) Receiver and oxidizer pressures.

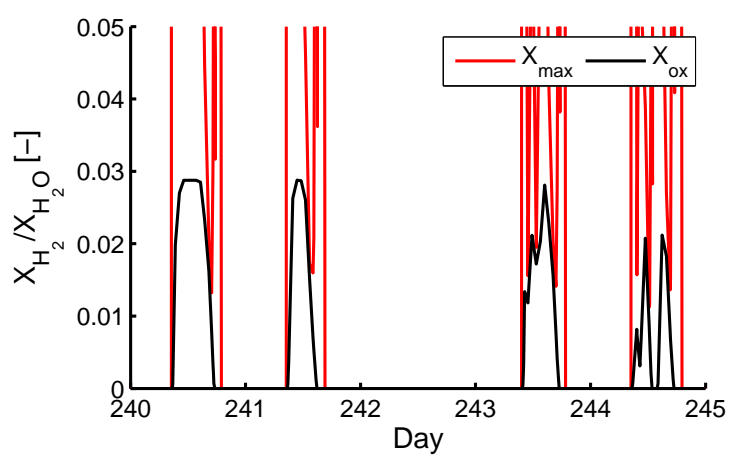

(f) Relation between hydrogen and water mass fractions inside the oxidizer.

Figure 4. Simulation details of 5 days.

days 243 and 244 the peak power barely reached $1 \mathrm{MW}$. Figure 4(c) shows the $\mathrm{CeO}_{2}$ temperatures inside the receiver and the oxidizer. The operating reduction temperature is ranged between $1850-1950^{\circ} \mathrm{C}$ and the operating oxidation temperature is around $1000-1100{ }^{\circ} \mathrm{C}$. When the plant is shut down, the temperature decreased quickly and 32 hours after is close to the ambient temperature. The pressure inside both reactors is shown in Figure 4(d). While the receiver works at ambient pressure, the oxidizer nominal pressure has been set to 1.5 bar in order to assure higher pressure than ambient when the hydrogen production is large. The degree of reduction is depicted in Figure 4(e), where peaks of 0.25 can be observed. For achieving the total oxidation of the $\mathrm{CeO}_{2}$, a minimum amount of water per hydrogen released is required inside the ox- idizer (Bulfin et al., 2015). In Figure 4(f) is depicted as $x_{\max }$ (the mass fraction of hydrogen into water) and it limits the amount of hydrogen produced at the oxidizer with the water used. The figure shows that in the whole simulation, the hydrogen released $\left(x_{o x}\right)$ is lower than the maximum amount expected at the equilibrium.

\section{Conclusions}

In this work, a dynamic model of a solar hydrogen plant based on the $\mathrm{CeO}_{2}$ redox cycle has been presented. The model has been developed with an object-oriented modelling methodology that it allows the re-used of several work previously developed. The system is design to study the transient behaviour of the plant in annual simulations. It was implemented with the Modelica language and sim- 
ulated with Dymola 2017. A basic automatic control system based on on-off controllers to guarantee the system stability was included.

The model predictions are reasonable and some usual simulation problems like zero-mass flows were solved with effectiveness. The computational effort of the model is low, therefore it can be used in optimization and control studies.

Increasing the model accuracy should be the objective of next works. The model reveals that thermal inertia of the $\mathrm{CeO}_{2}$ is too much high to accomplish the oxidation without extract heat flow from the reactor. The results suggest to review the assumptions related with heat losses and design a cooling systems at the oxidizer. The optimization of the plant through few operating parameters has demonstrated the flexibility of the system to be improved. Future studies should include operating cost and advanced operating strategies.

\section{Acknowledgement}

This work was performed as part of the ASTRI, a project supported by the Australian Government, through the Australian Renewable Energy Agency (ARENA).

\section{Appendix I: Model parameters}

\section{References}

Stéphane Abanades, Patrice Charvin, Gilles Flamant, and Pierre Neveu. Screening of water-splitting thermochemical cycles potentially attractive for hydrogen production by concentrated solar energy. Energy, 31:2469-2486, 2006. doi:10.1016/j.energy.2005.11.002.

Simon Ackermann and Aldo Steinfeld. Diffusion of oxygen in Ceria at elevated temperatures and its application to $\mathrm{H} 2 \mathrm{O} / \mathrm{CO} 2$ splitting thermochemical redox cycles. The Journal of Physical Chemistry Cournal of, 118, 2014.

AUSTELA. The NREL System Advisor Model for Australian CSP Stakeholders (SAM), 2016. URL http://www.austela.org.au/projects.

Roman Bader, Luke J. Venstrom, Jane H. Davidson, and Wojciech Lipiński. Thermodynamic analysis of isothermal redox cycling of ceria for solar fuel production. Energy and Fuels, 27(9):5533-5544, 2013. doi:10.1021/ef400132d.

Manuel Blanco-Muriel, Diego C. Alarcón-Padilla, Teodoro López-Moratalla, and Martín Lara-Coira. Computing the solar vector. Solar Energy, 70(5):431-441, 2001. doi:10.1016/S0038-092X(00)00156-0.

B. Bulfin, F. Call, M. Lange, O. Lübben, C. Sattler, R. Pitz-Paal, and I. V. Shvets. Thermodynamics of $\mathrm{CeO} 2$ thermochemical fuel production. Energy and Fuels, 29(2):1001-1009, 2015. doi:10.1021/ef5019912.

William C Chueh, Christoph Falter, Mandy Abbott, Danien Scipio, Philipp Furler, Sossina M Haile, and Aldo Steinfeld. High-flux solar-driven thermochemical dissociation of $\mathrm{CO} 2$ and $\mathrm{H} 2 \mathrm{O}$ using nonstoichiometric ceria. Sci-

\begin{tabular}{|c|c|}
\hline \multicolumn{2}{|l|}{ Heliostat field } \\
\hline Start-up power: & $0.6 \mathrm{MW}$ \\
\hline Shutdown power: & $0.3 \mathrm{MW}$ \\
\hline Deploy angle: & $8^{\circ}$ \\
\hline \multicolumn{2}{|l|}{ Ceria pump } \\
\hline Mass flow rate: & $2.5 \mathrm{~kg} / \mathrm{s}$ \\
\hline Shutdown Temperature: & $1000^{\circ} \mathrm{C}$ \\
\hline \multicolumn{2}{|c|}{ Ceria Heat exchanger } \\
\hline Heat transfer coefficient: & $500 \mathrm{~W} /\left(\mathrm{m}^{2} \mathrm{~K}\right)$ \\
\hline \\
\hline Reactor mass: & $500 \mathrm{~kg}$ \\
\hline Diameter: & $0.67 \mathrm{~m}$ \\
\hline Lenght: & $0.34 \mathrm{~m}$ \\
\hline Maximum $\mathrm{CeO}_{2}$ volume: & $25 \%$ \\
\hline Emitance: & 0.88 \\
\hline $\begin{array}{l}\text { Convective coefficient: } \\
\text { Oxidizer }\end{array}$ & $10 \mathrm{~W} /\left(\mathrm{m}^{2} \mathrm{~K}\right)$ \\
\hline Reactor mass: & $500 \mathrm{~kg}$ \\
\hline Diameter: & $0.67 \mathrm{~m}$ \\
\hline Lenght: & $0.34 \mathrm{~m}$ \\
\hline Maximum $\mathrm{CeO}_{2}$ volume: & $25 \%$ \\
\hline $\begin{array}{r}\text { Convective coefficient: } \\
\text { Tank }\end{array}$ & $500 \mathrm{~W} /\left(\mathrm{m}^{2} \mathrm{~K}\right)$ \\
\hline Diameter: & $0.5 \mathrm{~m}$ \\
\hline Height: & $0.5 \mathrm{~m}$ \\
\hline $\begin{array}{l}\text { Convective coefficient: } \\
\qquad N_{2} \text { Source }\end{array}$ & $10 \mathrm{~W} /\left(\mathrm{m}^{2} \mathrm{~K}\right)$ \\
\hline Gas Mass flow rate: & $0.25 \mathrm{~kg} / \mathrm{s}$ \\
\hline Shutdown temperature: & $700{ }^{\circ} \mathrm{C}$ \\
\hline
\end{tabular}

\begin{tabular}{|c|c|}
\hline \multicolumn{2}{|c|}{$N_{2}$ valve } \\
\hline Design pressure drop: & $0.05 \mathrm{bar}$ \\
\hline Closing pressure drop: & 0.025 bar \\
\hline \multicolumn{2}{|c|}{$N_{2}$ Heat exchanger } \\
\hline Heat transfer coefficient: & $500 \mathrm{~W} /\left(\mathrm{m}^{2} \mathrm{~K}\right)$ \\
\hline Area of exchange: & $5 \mathrm{~m}^{2}$ \\
\hline \multicolumn{2}{|c|}{$\mathrm{H}_{2} \mathrm{O}$ Source } \\
\hline Gas Mass flow rate: & $0.71 \mathrm{~kg} / \mathrm{s}$ \\
\hline $\mathrm{H}_{2} \mathrm{O}$ Mass fraction: & 0.45 \\
\hline Shutdown temperature: & $400{ }^{\circ} \mathrm{C}$ \\
\hline \multicolumn{2}{|c|}{$\mathrm{H}_{2} \mathrm{O}$ valve } \\
\hline Design pressure drop: & 0.5 bar \\
\hline Closing pressure drop: & $0.25 \mathrm{bar}$ \\
\hline \multicolumn{2}{|c|}{$\mathrm{H}_{2}$ Heat exchanger } \\
\hline Heat transfer coefficient: & $500 \mathrm{~W} /\left(\mathrm{m}^{2} \mathrm{~K}\right)$ \\
\hline Area of exchange: & $5 \mathrm{~m}^{2}$ \\
\hline
\end{tabular}


ence (New York, N.Y.), 330(6012):1797-801, dec 2010. doi:10.1126/science.1197834.

Dassault Systemes. Dymola 2017 - Dynamic Modeling Laboratory, 2016. URL www. 3ds. com.

Alberto de la Calle, Jim Hinkley, Paul Scott, and John Pye. SolarTherm : A New Modelica Library and Simulation Platform for Concentrating Solar Thermal Power Systems. Proc. 9th EUROSIM Congress on Modelling and Simulation, pages 12, 2016a. doi:10.1109/EUROSIM.2016.162.

Alberto de la Calle, Lidia Roca, Javier Bonilla, and Patricia Palenzuela. Dynamic modeling and simulation of a double-effect absorption heat pump. International Journal of Refrigeration, 72:171-191, 2016b. doi:10.1016/j.ijrefrig.2016.07.018.

Lawrence D'Souza. Thermochemical hydrogen production from water using reducible oxide materials: a critical review. $M a$ terials for Renewable and Sustainable Energy, 2(1):7, feb 2013. doi:10.1007/s40243-013-0007-0.

John A. Duffie and William A. Beckman. Solar Engineering of Thermal Processes. Wiley, New York, USA, 4th edition, 2013. ISBN 9780470873663. doi:10.1002/9781118671603.

Ivan Ermanoski. Maximizing Efficiency in Two-step Solarthermochemical Fuel Production. Energy Procedia, 69:17311740, 2015. doi:10.1016/j.egypro.2015.03.141.

Ivan Ermanoski, Nathan P. Siegel, and Ellen B. Stechel. A New Reactor Concept for Efficient Solar-Thermochemical Fuel Production. Journal of Solar Energy Engineering, 135 (3):031002, 2013. doi:10.1115/1.4023356.

Edward A. Fletcher. Solarthermal Processing: A Review. Journal of Solar Energy Engineering, 123(May 2001):63, 2001. doi:10.1115/1.1349552.

Philipp Furler, Jonathan R. Scheffe, and Aldo Steinfeld. Syngas production by simultaneous splitting of $\mathrm{H} 2 \mathrm{O}$ and $\mathrm{CO} 2 \mathrm{via}$ ceria redox reactions in a high-temperature solar reactor. Energy \& Environmental Science, 5(3):6098, 2012. doi:10.1039/c1ee02620h.

Xiang Gao, Alejandro Vidal, Alicia Bayon, Roman Bader, Jim Hinkley, Wojciech Lipiski, and Antonio Tricoli. Efficient ceria nanostructures for enhanced solar fuel production: Via high-temperature thermochemical redox cycles. Journal of Materials Chemistry A, 4(24):9614-9624, 2016. doi:10.1039/c6ta02187e.

IRENA and IEA-ETSAP. Technology Brief 4: Thermal Storage. Technical Report January, 2013.

Jiyong Kim, Terry a. Johnson, James E. Miller, Ellen B. Stechel, and Christos T. Maravelias. Fuel production from CO2 using solar-thermal energy: system level analysis. Energy \& Environmental Science, 5(9):8417, 2012. doi:10.1039/c2ee21798h.

Nathan S Lewis and Daniel G Nocera. Powering the planet: Chemical challenges in solar energy utilization. PNAS, 104 (42):15729-15735, 2007.
Bonnie J. McBride, Michael J. Zehe, and Sanford Gordon. NASA Glenn Coefficients for Calculating Thermodynamic Properties of Individual Species. Technical Report NASA/TP-2002-211556, National Aeronautics and Space Administration (NASA), Cleveland OH, USA, 2002.

Modelica Association. Modelica Specification 3.3, 2016. URL www.modelica.org/documents.

Christopher L. Muhich, Brian D. Ehrhart, Ibraheam Al-Shankiti, Barbara J. Ward, Charles B. Musgrave, and Alan W. Weimer. A review and perspective of efficient hydrogen generation via solar thermal water splitting. Wiley Interdisciplinary Reviews: Energy and Environment, pages n/a-n/a, 2015. doi:10.1002/wene.174.

NREL. The Solar Power Tower Integrated Layout and Optimization Tool (SolarPILOT), 2016. URL http://www.nrel.gov/csp/solarpilot.html.

J. O'Gallagher and R. Winston. Development of compound parabolic concentrators for solar energy. International Journal of Ambient Energy, 4(4):171-186, oct 1983. doi:10.1080/01430750.1983.9675885.

Hans Olsson, Martin Otter, Sven Erik Mattsson, and Hilding Elmqvist. Balanced Models in Modelica 3.0 for Increased Model Quality. In Proc. 6th International Modelica Conference, pages 21-33, Bielefeld, Germany, 2008.

Linda R. Petzold. A description of DASSL: a Diferential/Algebraic System Solver. Scientific Computing, pages 65-68, 1983.

Robert Pitz-Paal, Nicolas Bayer Botero, and Aldo Steinfeld. Heliostat field layout optimization for high-temperature solar thermochemical processing. Solar Energy, 85(2):334-343, 2011. doi:10.1016/j.solener.2010.11.018.

R. Ramachandran and R. K. Menon. An overview of industrial uses of hydrogen. International Journal of Hydrogen Energy, 23(7):593-598, 1998. doi:10.1016/S0360-3199(97)00112-2.

Martin Roeb, Martina Neises, Nathalie Monnerie, Friedemann Call, Heike Simon, Christian Sattler, Martin Schmücker, and Robert Pitz-Paal. Materials-Related Aspects of Thermochemical Water and Carbon Dioxide Splitting: A Review. Materials, 5(12):2015-2054, oct 2012. doi:10.3390/ma5112015.

Jonathan R. Scheffe and Aldo Steinfeld. Thermodynamic Analysis of Cerium-Based Oxides for Solar Thermochemical Fuel Production. Energy \& Fuels, 26(3):1928-1936, mar 2012. doi:10.1021/ef201875v.

Z. S. Spakovszky. Unified Engineering: Thermodynamics and Propulsion, 2008. URL web. mit. edu/16. unified.

S. Wilcox and W. Marion. Users manual for TMY3 data sets. Technical Report NREL/TP-581-43156, The National Renewable Energy Laboratory (NREL), Golden CO, USA, 2008. 